\title{
La competencia plurilingüe y pluricultural en la formación de futuros docentes de lenguas extranjeras en una universidad pública en Colombia
}

\author{
The Plurilingual and Pluricultural Competence in Foreign Language \\ Pre-Service Teacher Training in a Colombian Public University
}

\author{
A competência plurilíngüe e pluricultural na formação de futuros \\ docendes de línguas estrangeiras numa universidade pública na Colômbia
}

Fabio Alberto Arismendi Gómez ${ }^{1}$

Resumen

Este artículo presenta una experiencia pedagógica en la cual se implementó un curso llamado Introducción al Plurilingüismo en la Licenciatura en Lenguas Extranjeras de la Universidad de Antioquia, Medellín, Colombia, con el fin de favorecer el desarrollo de la competencia plurilingüe y pluricultural de los futuros docentes de lenguas. Con miras a analizar el impacto de dicho curso en la formación de los estudiantes, se realizó un análisis sistemático de datos recogidos por medio de cuestionarios, un grupo focal y los textos producidos por los estudiantes durante las diferentes versiones del curso. Los hallazgos mostraron cambios en las representaciones de los estudiantes sobre el bilingüismo y el plurilingüismo. Además, se evidenció la pertinencia y necesidad de crear espacios de reflexión sobre este tipo de temas en la formación de docentes de lenguas. En la primera parte del texto, se expone el marco teórico. Luego, se describe la experiencia pedagógica y la forma en que se recogieron y analizaron los datos. Finalmente, se presentan algunos de los hallazgos de la experiencia y las conclusiones.

Palabras clave: Competencia plurilingüe y pluricultural, plurilingüismo, formación de docentes de lenguas

Abstract

This article presents a pedagogical experience in which a teacher implemented a course called Introduction to Plurilingualism, in the undergraduate foreign language teacher program at Universidad de Antioquia, Medellín, Colombia, in order to favor the development of plurilingual and pluricultural competence in future language teachers. In order to analyze the impact of that course on students' training, a systematic analysis was carried out from data obtained from questionnaires, a focus group, and the texts students wrote during the different versions of the course. Findings showed changes in students' representations about bilingualism and plurilingualism. In addition, the data showed the need and importance of creating spaces to reflect on this kind of topic in the context of teacher's training. Firstly, the theoretical framework is presented; then, the pedagogical experience and the way in which data were collected and analyzed. Finally, some of the findings of the experience and the conclusions are presented.

Keywords: Plurilingual and pluricultural competence, plurilingualism, foreign language teachers' training.

Resumo

Este artigo apresenta uma experiência educacional em que um curso chamado Introdução ao plurilingüismo na Licenciatura de Línguas Estrangeiras da Universidade de Antioquia, Medellín, Colômbia, foi implementado a fim de promover o desenvolvimento da competência plurilíngüe e pluricultural dos futuros professores da língua. A fim de analisar o impacto do curso na formação dos alunos, uma análise sistemática dos dados coletados por meio de questionários, grupo de foco, e textos produzidos pelos alunos durante as diferentes versões do curso. Os resultados mostraram alterações no desempenho dos alunos sobre bilinguismo e multilinguismo. Além disso, a relevância e a necessidade de criar espaços de reflexão sobre estas questões na formação de professores de línguas era evidente. Na primeira parte do texto, o quadro teórico é apresentado. Em seguida, a experiência educacional e a forma como os dados foram coletados e analisados são descritos. Finalmente, algumas das conclusões e achados da experiência são apresentados.

Palavras Chave: Competênia multilungue e multicultural, multilinguismo, formação de docentes de língua

Artículo recibido el 2 de abril de 2015 y aprobado el 27 de enero de 2016

1 Escuela de Idiomas, Universidad de Antioquia, Medellín, Colombia. Correo electrónico: fabio.arismendi@udea.edu.co. 
Universidad Pedagógica Nacional

Facultad de Humanidades

\section{Introducción}

Colombia es, según los artículos 7 y 10 de la Constitución Política de 1991, un país multiétnico y multicultural. De acuerdo con Landaburu (2012), existen alrededor de 68 lenguas habladas actualmente en el país. Aparte del castellano, lengua materna de la mayoría de la población, existen dos lenguas criollas: el criollo hablado en San Andrés y Providencia, y el palenquero hablado en San Basilio de Palenque; y alrededor de 65 lenguas indígenas pertenecientes a 21 estirpes distintas que son habladas por el $2 \%$ de la población ${ }^{2}$. También podemos contar el romaní, lengua de los pueblos gitanos presentes en el país (González de Pérez, 2012). A pesar de la gran riqueza lingüística y cultural que estas lenguas y sus hablantes representan para nuestro país, pareciera que muchos colombianos ignoramos esta diversidad. En lo que respecta a contextos diversos, Montagne-Macaire (2008, p. 30) señala que vivir en una sociedad multilingüe y multicultural implica desarrollar una "metacompetencia" que implica los siguientes saberes, aptitudes y actitudes:

1. Actitudes de aceptación positiva y de interés por la diversidad lingüística y cultural.

2. Saberes sobre las lenguas y las culturas.

3. Aptitudes para utilizar varias lenguas con fines comunicativos, comunicarse parcialmente en la ausencia de un código lingüístico común y saber identificar lenguas y culturas desconocidas.

A pesar de que dicha autora hace su propuesta en un contexto europeo, que difiere ampliamente del colombiano, se podría afirmar que las actitudes, saberes y aptitudes por desarrollar son válidos y necesarios en un país que dice ser multiétnico y multicultural.

\section{La formación de profesores de lenguas en el país y la diversidad lingüística}

La ley 1651 de 2013, al igual que los lineamientos de calidad para las licenciaturas en educación

2 Según Landaburu (2012) la población indígena total es aproximadamente el $3,4 \%$. Sin embargo, muchas comunidades perdieron sus lenguas originarias y solo el $2 \%$ de la población habla una lengua ancestral.
(MEN, 2014) y otros documentos emitidos por las alcaldías locales de algunas ciudades (por ejemplo, el acuerdo 089 de 2013 de la ciudad de Medellín) priorizan la enseñanza del inglés en los colegios públicos. Esta situación tiene sus antecedentes en 1994 cuando la nueva Ley General de Educación señaló como obligatoria la enseñanza de una sola lengua extranjera en los colegios y tuvo como consecuencia la desaparición en los currículos de otras lenguas, como el francés, que se enseñaba en muchos planteles educativos colombianos. A pesar de este panorama desfavorable para las lenguas extranjeras diferentes al inglés, aún existen en el país programas que forman docentes en otras lenguas. Así podríamos citar un programa de formación de profesores de alemán y uno de profesores de francés $^{3}$ exclusivamente, y alrededor de 18 programas $^{4}$ de pregrado que continúan formando profesores de inglés y de francés simultáneamente. Es el caso preciso de la Licenciatura en Lenguas Extranjeras de la Universidad de Antioquia, donde los estudiantes reciben el mismo número de horas de instrucción en ambas lenguas durante sus diez semestres de formación. Los estudiantes de este programa desarrollan durante sus estudios una competencia plurilingüe pues viven con al menos tres lenguas diferentes (una materna y dos extranjeras); además muchos de ellos deciden estudiar una tercera lengua extranjera en Multilingua, uno de los programas ofrecidos por la universidad 5 .

3 Se trata de los programas de la Universidad Nacional de Colombia, sede Bogotá.

4 Dichos programas se ofrecen en las siguientes universidades: Universidad de Caldas, Universidad del Quindío, Universidad Católica de Oriente (Rionegro), Universidad de la Salle (Bogotá y Medellín), Pontificia Universidad Javeriana, Universidad Libre, Universidad Tecnológica del Chocó, Universidad Pedagógica Nacional, Universidad Santiago de Cali, Universidad del Valle, Universidad Pedagógica y Tecnológica de Tunja, Universidad del Cauca, Universidad del Atlántico (inglés, francés y alemán), Universidad San Buenaventura de Cartagena, Universidad de Pamplona, Universidad de Nariño, Universidad Central del Valle (Tuluá) y Universidad de Antioquia.

5 Multilingua es un programa financiado por la rectoría de la Universidad que ofrece 400 horas de formación ( 5 niveles) en ocho lenguas diferentes: inglés, francés, portugués, italiano, chino, japonés, turco y alemán. El programa es ofrecido como un estímulo académico a los estudiantes que deseen aprender otro idioma y que tengan un promedio crédito mínimo de 3,8. 
A pesar de poseer un amplio repertorio verbal, que en términos de Gumperz (1989, citado por Moore 2006, p. 100) se refiere a todas las lenguas que un hablante puede usar en recepción y en producción en diferentes situaciones comunicativas, los estudiantes del programa de Licenciatura en Lenguas tienen pocas oportunidades de reflexionar sobre lo que implica ser un hablante de varias lenguas. Adicional a esto, muchos de los estudiantes no son conscientes de la riqueza lingüística y cultural presente en nuestro territorio y de la manera de integrarla en sus clases de lengua extranjera; parecieran por el contrario querer concentrarse más en el conocimiento de culturas extranjeras que en descubrir la propia diversidad presente en el país.

Por las razones expuestas anteriormente, diseñé un curso electivo llamado Introducción al Plurilingüismo, que se ha ofrecido desde hace cuatro años a los estudiantes de últimos semestres del programa de Licenciatura en Lenguas Extranjeras de la Universidad de Antioquia y cuyo objetivo es explorar y analizar algunos principios que fundamentan la didáctica del plurilingüismo y su papel en la enseñanza de las lenguas extranjeras. Se busca no solo conocer algunas teorías sobre plurilingüismo desarrolladas en otros contextos, con miras a que los estudiantes entiendan su propia situación lingüística y la del país, sino también contribuir al desarrollo de la competencia plurilingüe y pluricultural de los estudiantes por medio de actividades prácticas y reflexivas. Igualmente se busca analizar el rol del maestro de lenguas extranjeras con respecto a la diversidad lingüística.

Castellotti (2006) señala que una de las áreas que requiere mayor trabajo con respecto al desarrollo de la competencia plurilingüe es la formación de maestros, dado que, en la mayoría de países, los profesores se conciben como "especialistas de una lengua" y no como profesores de lenguas. Esta misma autora argumenta que si deseamos formar profesores en el plurilingüismo, no es suficiente con sensibilizarlos a la pluralidad; se requiere formarlos por medio de la pluralidad, viviendo el plurilingüismo por medio de actividades prácticas de formación. Al respecto,
Bernaus, Andrade, Kervran, Murkoswa y Trujillo (2007) desarrollaron un kit de formación en la dimensión plurilingüe y pluricultural dirigido a los profesores, el cual tiene como objetivo invitar a los docentes a reflexionar sobre temas como las lenguas, las culturas y la comunicación en general por medio de itinerarios de trabajo sobre las dimensiones personal, social y profesional.

Existen pocos trabajos documentados en el país sobre experiencias de inclusión de la diversidad lingüística en los programas de formación de maestros de lenguas extranjeras. Podríamos citar los trabajos de Tapiero (2014) y de Agudelo (2007). La primera autora analiza en su investigación la promoción de la diversidad en contextos de homogeneidad en la formación de maestros en la Universidad Francisco José de Caldas en Bogotá. Por su parte, el segundo autor describe la experiencia de creación de un curso llamado Lenguaje, Cultura y Diversidad, en la Universidad de Antioquia, en el mismo programa donde se llevó a cabo la propuesta documentada en el presente texto. La inquietud por este tema en la formación de docentes de lenguas también es de interés en otros contextos en Latinoamérica. Por ejemplo, con respecto a Argentina, Klett (2014) señala que la mayoría de estudiantes en formación como maestros de lenguas en ese país desconocen el patrimonio de más de doce lenguas autóctonas además de las lenguas de inmigración. La misma autora señala que la formación de maestros de lenguas se ha hecho de forma monolingüe; es decir, si se forman maestros de inglés toda la formación se hará en esa lengua, desconociendo el aporte que otras lenguas podrían tener a la formación. La autora propone algunas estrategias para favorecer el plurilingüismo en la formación, similares a las que se reportan en esta experiencia.

En este artículo, presento la experiencia de creación, montaje, implementación y evaluación de dicho curso. Comenzaré con la exploración de algunos fundamentos teóricos; luego explicaré la forma en que se desarrolló el curso y se analizaron los datos. Finalmente, presentaré algunos hallazgos derivados de esta experiencia. 
Universidad Pedagógica Nacional

Facultad de Humanidades

\section{Marco teórico}

\section{Bilingüismo y políticas lingüísticas en Colombia}

A lo largo de la historia de la enseñanza de lenguas, el término bilingüismo se ha estudiado desde perspectivas muy diferentes (Bloomfield, 1935; Grosjean, 1982, 2010; Macnamara, 1967). Para este trabajo, nos identificamos con la definición de bilingüismo propuesta por Grosjean (2010, p. 4), según la cual es bilingüe quien "usa dos o más lenguas (o dialectos) en su vida diaria"6. Se presenta así una visión funcional del término, la cual no implica un dominio equilibrado de dos lenguas, como lo proponía Bloomfield en los años treinta.

En Colombia, diversas publicaciones dan cuenta de los trabajos en esta área (ver por ejemplo Mejía [2006, 2004, 2002], Galindo y Moreno [2008], Herazo, Jerez y Lorduy [2012]). Mejía (2007) señala que en Colombia no existe consenso sobre lo que significa ser bilingüe para los diferentes actores educativos. Según la autora, se han visto dos tipos de bilingüismo, uno que se refiere a lenguas de prestigio como el inglés y el francés, asociadas generalmente a las élites; y otro, relacionado con las lenguas locales y asociado generalmente a pobreza, violencia y desplazamiento. Por su parte, Guerrero (2012, p. 46) explica que en América Latina el "mal llamado bilingüismo" se refiere al uso de dos lenguas. Insiste en que "hay que separar bilingüismo de educación bilingüe y por otro lado separar bilingüismo social de bilingüismo individual” (p. 46). Ordóñez (2012) aboga por el término "educación para el bilingüismo" e insiste en la importancia de la lengua materna en los procesos de aprendizaje llamados bilingües en contextos mayoritariamente monolingües como el nuestro.

Es importante señalar que muchos de los trabajos realizados en el país en esta área están directamente relacionados con las políticas lingüísticas que imperan en la actualidad en el territorio colombiano, en particular con el plan de mejoramiento de los niveles de dominio del inglés en todos los niveles

6 Traducción propia. del sistema educativo. Así, Miranda (2012) sostiene que las políticas del Gobierno colombiano buscan el desarrollo de un bilingüismo aditivo y no de un bilingüismo social. Al igual que Miranda, autores como Cárdenas (2006), Guerrero (2008), Sánchez y Obando (2008) y Valencia (2007), han cuestionado la implementación que el Gobierno ha hecho del programa nacional de bilingüismo. Por ejemplo, Mejía (2006, p. 153) señala que Colombia ha tenido una larga tradición de integración de lenguas extranjeras en el currículo escolar con el objetivo de desarrollar una visión pluralista del mundo. Esta visión se ha modificado en los últimos años con el énfasis particular que el Gobierno le ha dado a la lengua inglesa. En la misma dirección, González de Zárate (2007) explica que las políticas lingüísticas del Gobierno solo favorecen la enseñanza del inglés, lo cual tiene como consecuencia privar "a nuestros niños del aprendizaje plurilingüe que tienen otros niños del mundo" (p. 59).

Además, González (2009), Guerrero (2008, 2009), Sánchez y Obando (2008) y Usma (2009), entre otros, han criticado la falta de claridad de las políticas lingüísticas del Gobierno, por ejemplo, en lo que concierne a las lenguas indígenas del país. Al respecto, Guerrero (2009) explica cómo las lenguas nativas, indígenas y criollas, no han sido valoradas en las políticas lingüísticas del país y cómo las nuevas políticas de fortalecimiento del inglés siguen desconociendo nuestra riqueza lingüística.

\section{Plurilingüismo, multilingüismo, competencia plurilingüe y pluricultural}

Un grupo de investigadores europeos ha desarrollado lo que algunos autores, entre ellos Candelier (2008), han llamado "didáctica del plurilingüismo". Desde esta perspectiva, se puede definir el plurilingüismo como la capacidad que tiene un individuo de utilizar "oportunamente diversas variedades lingüísticas"7 (Riley, 2003, p. 13). Esta definición se puede contrastar con la de multilingüismo, la cual, según el mismo autor y el Instituto Cervantes (2002), se refiere a la presencia de variedades lingüísticas en

7 Traducción propia. 
un mismo territorio mas no al dominio individual de otras lenguas. En este sentido, podríamos decir que Colombia tiene algunos territorios multilingües y que existen individuos plurilingües en territorios monolingües.

En el marco de la educación para el plurilingüismo, desde los años noventa, un amplio grupo de investigadores europeos ha trabajado en la definición del concepto de competencia plurilingüe y pluricultural, que aparece finalmente publicada en el Marco de Referencia Europeo y se define así:

La competencia plurilingüe y pluricultural hace referencia a la capacidad de utilizar las lenguas para fines comunicativos y de participar en una relación intercultural en que una persona, en cuanto agente social, domina - con distinto grado- varias lenguas y posee experiencia de varias culturas. Esto no se contempla como la superposición o yuxtaposición de competencias diferenciadas, sino como la existencia de una competencia compleja e incluso compuesta que el usuario puede utilizar. (Instituto Cervantes, 2002, p. 167)

Según Castellotti y Moore (2011, p. 243) ${ }^{8}$, este concepto implica tres características:

a. [L]a relación entre las dimensiones lingüística y cultural es afirmada como intrínseca e indisociable.

b. [S]e afirma, desde el punto de vista del aprendizaje de las lenguas, una lógica socio-identitaria, fundada en una experiencia de contacto y de alteridad y anclada en una trayectoria familiar, comunitaria y social.

c. [S]e rechaza la idea del nativo como hablante ideal o "perfecto bilingüe".

Dentro de esta perspectiva, Coste (2010) precisa que aprender varias lenguas no significa acumular competencias homogéneas y en compartimentos separados sino más bien que las lenguas y culturas están relacionadas y crean un repertorio lingüístico en el hablante. Al respecto, los autores del Marco de

8 Traducción propia
Referencia Europeo explican que la competencia plurilingüe incluye el conjunto de las lenguas que un individuo conoce sin tratarse en ningún momento de un "supuesto equilibrio" entre L1/L2. Además destacan las dimensiones pluriculturales de dicha competencia (Instituto Cervantes, 2002).

\section{Los enfoques plurales}

Candelier (2008) señala que dentro de la didáctica del plurilingüismo se han desarrollado algunos enfoques plurales que buscan, entre otros, desarrollar la competencia plurilingüe y pluricultural de los aprendices. Se les denomina plurales porque intentan abordar al mismo tiempo diferentes variedades lingüísticas y culturales. Aunque algunos de ellos no han sido lo suficientemente reconocidos o aceptados dentro de los currículos en el contexto europeo, existen muchas investigaciones y trabajos que dan cuenta de los resultados positivos de la implementación de dichos enfoques en las aulas. Estos enfoques son: la intercomprensión entre familias de lenguas, el despertar a las lenguas, la didáctica integrada y la educación intercultural. Dada la complejidad y amplitud del tema me limitaré en este artículo a proveer una breve definición de cada uno de ellos. La intercomprensión y el despertar a las lenguas son los enfoques más recientes, mientras que la educación integrada y la educación intercultural tienen ya una larga tradición en el contexto europeo.

\section{La intercomprensión}

Nació en los años ochenta (Escudé y Janin, 2010) y puede definirse como "comprender la lengua del otro y hacerse entender"9 (Degache, 2006, p. 21). Se trata entonces de un enfoque que busca evitar el uso de una lengua vehicular, que en la mayoría de los casos sería el inglés, como medio de comunicación en ausencia de un código lingüístico común. Por ejemplo, un colombiano y un brasileño cuyas lenguas maternas sean el español y el portugués respectivamente podrían, siguiendo el principio de la intercomprensión, comunicarse cada uno hablando en su lengua materna sin necesidad de

9 Traducción propia. 
Universidad Pedagógica Nacional

Facultad de Humanidades

tener conocimientos avanzados de la lengua del otro y sin necesidad de recurrir a una lengua vehicular como el inglés. Esto es posible gracias a la similitud lingüística de las lenguas que provienen de la misma familia. Sin embargo, para que el principio de la intercomprensión funcione se requiere un entrenamiento, el cual se puede realizar por medio de textos, programas o intercambios en línea con hablantes reales, como es el caso de la plataforma Galanet (véase Arismendi, 2011). A pesar de que es un tema poco estudiado en Latinoamérica, publicaciones recientes, como la de Tassara y Villalón (2014), dan cuenta de un inicio de exploración del tema.

\section{El despertar a las lenguas}

Es un enfoque trabajado particularmente con niños de preescolar o escuela primaria. Según Candelier (2006), este enfoque no busca la enseñanza de una lengua en particular sino tratar la diversidad lingüística como objeto de actividades pedagógicas. Por medio de actividades estructuradas, el maestro busca la reflexión y el desarrollo de una conciencia lingüística en sus estudiantes con respecto a lenguas que la escuela no enseña. Algunas investigaciones (Montagne-Macaire, 2008; Moore y Castellotti, 2001; Simon, 2005) han mostrado que utilizar este enfoque puede favorecer la descentración de los aprendices, y actitudes de apertura e interés hacia el otro y su(s) lengua(s). En Colombia, el trabajo de Rengifo (2011), realizado durante su práctica académica de pregrado de la Universidad del Valle en Cali, da cuenta del impacto positivo de la implementación de actividades de despertar a las lenguas en contexto "monolingüe".

\section{La didáctica integrada}

Se refiere, en términos de Candelier (2008), a la forma en que la escuela europea ha organizado la enseñanza de lenguas: lengua materna, lengua extranjera (1), lengua extranjera (2). El mismo autor señala que los usos más recientes del término no distinguen la forma en que se enseñan las lenguas, es decir que se puede referir a la enseñanza de la lengua en sí o a la enseñanza de otras áreas en la lengua.

\section{La educación intercultural}

Difiere ligeramente de los enfoques anteriores en cuanto que no es un enfoque lingüístico, pues no busca directamente la manipulación de la lengua. Es uno de los enfoques más antiguos. Según Candelier (2008) tiene una tradición de aproximadamente cuarenta años, por lo menos desde el punto de vista de sus fundamentos. Montagne-Macaire (2008) explica que el enfoque de las relaciones interculturales no busca "una situación única exitosa" sino que "admite la posibilidad de 'no comprensión' que interroga las culturas y las compromete a remediaciones diversas, de las cuales la escuela podría apoderarse para promoverlas"10 (p. 30).

\section{La identidad}

Una de las nociones centrales trabajadas en el desarrollo de la competencia plurilingüe y pluricultural es la identidad. En efecto, se trata de un término bastante complejo y que ha sido abordado desde muchas perspectivas. Según Kastersztein (1990) la identidad es "una estructura polimorfa, dinámica, cuyos elementos constitutivos son los aspectos psicológicos y sociales ligados a la situación relacional en un momento dado de un agente social (individuo o grupo) como actor social"11 (p. 28). Lagarde (2008) explica la complejidad del término y propone, retomando a los filósofos Gilles Deleuze y Félix Guattari, la metáfora de la identidad como raíz y como rizoma. La identidad como raíz sugiere una interpretación reductora y simplista en la que esta se percibe como una sola, mientras que la identidad rizoma explica las diferentes facetas que puede tener la identidad; estas pueden incluso ser incoherentes, pero complementarias. Esta segunda perspectiva es la que adopto para este trabajo. En este sentido, la identidad es el producto de un proceso dinámico y puede tener diferentes componentes plurales. Lagarde (2008) explica también que el término implica distintas dimensiones, como la identidad personal, la social, la cultural y la lingüística.

\footnotetext{
10 Traducción propia.
}

11 Traducción propia. 
Con respecto a la categoría de identidad lingüística, es importante señalar que la relación entre lengua e identidad, desde una lectura socioconstructivista, permite definir las identidades individuales como "dinámicas, complejas, difusas y plurales” (Sabatier, 2010, p. 134). Además, la identidad lingüística y las actitudes lingüísticas se modifican permanentemente (García, 2014, p. 147). Thomas (2004, citado por García 2014) explica que "el uso de la lengua puede indicar los cambios de identidad o la forma en que un sujeto se considera miembro de un grupo lingüístico, es decir, su identidad etnolingüística” (p. 151).

Para concluir esta sección, recordemos, como lo explica Klett (2011), que "situarse en la interfaz identitaria es entonces aceptar el encuentro de la identidad primaria (nuestra filiación genética, social, grupal, cultural) y aquella que se establece por intermedio de la nueva lengua" (p. 3). La autora señala que dicho proceso puede ser conflictivo pero debería permitirnos no ver "la diferencia como una amenaza" (p. 3). Por el contrario, retomando a Charaudeau (2006), Klett (2011) arguye que dicha confrontación debería ayudar al sujeto a "reconstruir su identidad afianzándola”.

\section{Experiencia pedagógica}

\section{Contexto de enseñanza}

Los estudiantes de la Licenciatura en Lenguas Extranjeras de la Universidad de Antioquia deben matricular en los tres últimos semestres de su programa tres cursos electivos en Inglés o en Francés de cuatro créditos cada uno (64 horas presenciales y 80 de trabajo independiente) para un total de 12 créditos ${ }^{12}$. En la actualidad, el programa cuenta con un banco de aproximadamente 10 electivas que se ofrecen alternadamente entre los semestres uno y dos de cada año con el fin de permitir el acceso a diferentes estudiantes y de garantizar un número mínimo de inscritos para poder desarrollar el curso.

12 En el año 2011, según directrices del Ministerio de Educación, se realizó una reforma a los créditos. Por lo tanto, el curso tiene actualmente tres créditos pero el mismo número de horas.
Dado el número de electivas, los estudiantes eligen aquellas que más les interesan y en la lengua de su preferencia. El curso Introducción al Plurilingüismo se creó y ofreció por primera vez en el año 2011. La lengua de instrucción del curso en todas sus versiones ha sido el francés. Hasta el momento se ha ofrecido cuatro veces, con el siguiente número de estudiantes:

Tabla 1 semestres en los que se ha ofrecido el curso y número de estudiantes

\begin{tabular}{|c|c|}
\hline SEMESTRE & NÚMERO DE ESTUDIANTES \\
\hline $2011--I$ & 9 \\
\hline $2012--$ & 12 \\
\hline $2013-I$ & 19 \\
\hline $2014--$ & 25 \\
\hline
\end{tabular}

Merece la pena señalar que el número de estudiantes interesados en el curso se ha incrementado desde que este se ofreció por primera vez en 2011, y alcanzó su cupo máximo en el semestre 2014-I. El objetivo general del curso es explorar y analizar algunos principios que fundamentan la didáctica del plurilingüismo y su papel en la enseñanza de las lenguas extranjeras. Dentro de los objetivos específicos del curso incluimos:

a. Analizar de manera crítica las nociones de monolingüismo, bilingüismo, multilingüismo y plurilingüismo y su pertinencia en el contexto colombiano.

b. Reconocer la pluralidad lingüística y cultural de nuestro propio país.

c. Explorar los enfoques plurales y analizar de manera crítica su pertinencia en el medio colombiano.

d. Participar en una experiencia de intercomprensión en la plataforma Galanet.

Es importante mencionar que si bien se crearon unas unidades de trabajo y se escogieron unos contenidos precisos en el 2011, estos se han ido ajustando a los intereses y necesidades de los nuevos grupos de estudiantes. La organización del curso se presenta en la tabla 2 . 
Universidad Pedagógica Nacional

Facultad de Humanidades

Tabla 2. Organización y contenido del curso

\begin{tabular}{|c|c|c|}
\hline UNIDAD & NOMBRE & CONTENIDOS ESPECÍFICOS \\
\hline 1 & $\begin{array}{l}\text { Las lenguas } \\
\text { en el mundo y } \\
\text { el repertorio } \\
\text { lingüístico de } \\
\text { los estudiantes }\end{array}$ & $\begin{array}{l}\text { Qué es una lengua, las lenguas } \\
\text { del mundo, las representacio- } \\
\text { nes de los estudiantes sobre } \\
\text { las lenguas, biografías lingüísti- } \\
\text { cas de los estudiantes }\end{array}$ \\
\hline 2 & $\begin{array}{l}\text { Marco } \\
\text { conceptual }\end{array}$ & $\begin{array}{l}\text { Análisis de diferentes perspec- } \\
\text { tivas sobre bilingüismo, multilin- } \\
\text { güismo, plurilingüismo, compe- } \\
\text { tencia plurilingüe }\end{array}$ \\
\hline 3 & $\begin{array}{l}\text { La situación } \\
\text { lingüística en } \\
\text { Colombia }\end{array}$ & $\begin{array}{l}\text { Exploración de la pluralidad } \\
\text { lingüística y cultural colombia- } \\
\text { na presente en sus diferentes } \\
\text { lenguas y comunidades : palen- } \\
\text { quero, criollo, lenguas indíge- } \\
\text { nas, romaní }\end{array}$ \\
\hline 4 & La identidad & $\begin{array}{l}\text { Diferentes aproximaciones a la } \\
\text { identidad. La identidad cultural } \\
\text { y la identidad lingüística }\end{array}$ \\
\hline 5 & $\begin{array}{l}\text { Los enfoques } \\
\text { plurales }\end{array}$ & $\begin{array}{l}\text { Acercamiento a la intercom- } \\
\text { prensión, el despertar a las len- } \\
\text { guas y el enfoque intercultural } \\
\text { Participación en una sesión de } \\
\text { la plataforma Galanet }\end{array}$ \\
\hline
\end{tabular}

Para cada una de las versiones del curso se recurrió a invitados con el fin de profundizar en algunos aspectos, por ejemplo un profesor y un grupo de estudiantes indígenas pertenecientes a diferentes programas de la universidad; un profesor que realizó una clase en catalán con el objetivo de analizar la noción de intercomprensión, entre otros. Con el grupo del semestre 2013-I tuvimos la oportunidad de realizar una visita a una institución educativa indígena ubicada en el municipio de Jardín, a 138 kilómetros de la ciudad.

Paralelo al desarrollo del curso presencial, los estudiantes estaban inscritos en una sesión de trabajo en la plataforma Galanet, con el objetivo de vivir una experiencia real de intercomprensión en lenguas romances ${ }^{13}$. Siguiendo las ideas de Castellotti (2006), el acercamiento al plurilingüismo se concibió no solo por medio de la teoría sino también por medio de actividades prácticas que ejemplificaban los diferentes enfoques presentados.

13 Por limitaciones de espacio, en este artículo no se documenta en detalle el análisis de la utilización de la plataforma Galanet durante el curso.

\section{Recolección y análisis de datos}

Con miras a evaluar la pertinencia y el impacto del curso, realicé un análisis sistemático. Me interesaba saber cómo había impactado el curso los puntos de vista de los estudiantes, sus representaciones sobre el tema y su formación como futuros docentes. La recolección de datos incluyó las siguientes fuentes: 1. Los trabajos escritos por los estudiantes, entre los cuales se cuentan sus biografías lingüísticas y algunos textos de reflexión sobre los temas abordados en el curso; 2. Un grupo focal (Dendinger, 2000) que se llevó a cabo en el año 2011 al finalizar la primera versión del curso y en el cual participaron siete de los nueve estudiantes matriculados en dicho semestre. 3. Cuestionarios semiestructurados (Albarello, 2003; Van der Maren, 2003) realizados en el año 2013 y que fueron dirigidos a los estudiantes que habían tomado el curso en los años 2012 y 2013. Dichos cuestionarios fueron piloteados y posteriormente implementados utilizando la herramienta de Googledocs; se recibieron en total 19 respuestas. 4. Las evaluaciones del curso y del profesor realizadas por el programa al final de cada semestre. Para analizar dichos datos, recurrí a la creación de categorías previas y emergentes por medio de un análisis de contenido (Van der Maren, 2003).

\section{Hallazgos y discusión}

La experiencia de dictar este curso durante cuatro años y el análisis sistemático de los datos recogidos durante este tiempo me permitieron develar cambios en las representaciones de los estudiantes sobre la valoración de sus propios repertorios lingüísticos e igualmente descubrir algunos logros en los planos personal y académico.

\section{Cambio en las representaciones}

\section{De bilingüismo a plurilingüismo}

El primer contacto con los diferentes grupos de estudiantes me permitió constatar que la mayoría de ellos tenían una concepción de bilingüismo muy cercana a la propuesta por Bloomfield (1935), según la cual un bilingüe es quien domina dos lenguas 
perfectamente, al nivel de un nativo, y alejada de concepciones más contemporáneas sobre el dominio de varias lenguas. Con respecto a la palabra plurilingüe, muchos aseguraron no saber con claridad a qué se refería, otros la asociaban simplemente al hecho de ser políglota, de hablar varias lenguas. Dichas representaciones les impedían valorar sus amplios repertorios lingüísticos y su competencia plurilingüe pues consideraban que no tenían el nivel apropiado para considerarse bilingües o plurilingües.

Ahora bien, una de las primeras tareas del curso consistió en elaborar una biografía lingüística. Este ejercicio permitió a todos los estudiantes y a mí como profesor darnos cuenta del inmenso repertorio lingüístico de ellos. Todos hablaban español como lengua materna, e inglés y francés como lenguas extranjeras. Aparte de esto, muchos habían estudiado otras lenguas extranjeras, como alemán, italiano, portugués, chino, japonés o turco en el programa Multilingua. Aun para sorpresa de muchos, algunos estudiantes por razones variadas habían estudiado griego, latín o hebreo. La reflexión sobre sus propias biografías lingüísticas y la exploración de la noción de competencia plurilingüe les permitieron comprender que no es necesario dominar las otras lenguas al nivel de un nativo para considerarse como poseedor de una competencia plurilingüe. Los siguientes testimonios ejemplifican esta evolución en las representaciones

Ahora veo el plurilingüismo como una cuestión de uso, no de perfecta utilización gramatical y fluidez en los idiomas. Además pienso que es darle la misma importancia a todas las lenguas. (Manuel, cuestionario, 06/08/2013) (14 $^{14}$

Antes con la relación que encontraba con ser políglota, la idea era que había un dominio de las lenguas cercano a la perfección o a los nativos. (Felipe, cuestionario, 23/08/2013)

14 Con miras a proteger la identidad de los participantes, se utilizan seudónimos en los testimonios. Los textos no presentan corrección alguna. Entre paréntesis se señala el instrumento de recolección de donde proviene el testimonio y la fecha en que se obtuvo.
[El curso] creó en mi [sic] una conciencia que me permite valorar cualquiera de mis conocimientos en cada idioma que hablo, sé que no debo ser C2 en todos. (Camilo, cuestionario, 13/08/2013)

Estos testimonios reflejan una apropiación del concepto de competencia plurilingüe expuesto en el marco teórico, según el cual las lenguas no se acumulan en compartimentos separados y de manera homogénea sino por el contrario se interrelacionan de manera compleja interactuando entre sí (Coste, 2010). Además, la elaboración de las biografías lingüísticas impulsó a los estudiantes a reflexionar sobre las diferentes relaciones que establecen con las lenguas que hablan y estudian e incluso a superar dificultades, como lo plantea Claudia:

[al realizar mi biografía] me empecé a encontrar con cosas que tenía guardadas, muy guardadas [...] y entendí mi bloqueo con el inglés. (Claudia, grupo focal, 27/02/2012)

\section{Sobre las motivaciones}

También se presenta un cambio con respecto a las motivaciones iniciales hacia el curso. La mayoría de los estudiantes encuestados afirmaron tomar el curso porque el horario les convenía. En menor medida, algunos señalaron haberlo hecho porque conocían al profesor, porque el curso era en francés y deseaban practicar dicha lengua o porque el nombre del curso les llamaba la atención a pesar de no tener conocimientos sobre el tema. Cabe anotar que algunos estudiantes no se encontraban motivados hacia la lengua francesa y se negaron incluso a hablar francés en las primeras sesiones de clase porque consideraban que no tenían un nivel apropiado. Después de reflexionar sobre sus biografías lingüísticas y valorar los pocos conocimientos que tenían en la lengua francesa, estos estudiantes se expresaron en dicha lengua. La motivación inicial de todos cambió o aumentó, y mostraron un interés bastante alto en las temáticas del curso, como lo muestra el siguiente testimonio:

Al comienzo no tenía más opción porque era el único curso que me servía según mi horario, pero yo no quería coger ningún curso en francés. Sin 
Universidad Pedagógica Nacional

Facultad de Humanidades

embargo, el curso se convirtió en una experiencia muy motivadora y gratificante para mí porque pude aprender de otras culturas y conocer más sobre la historia de mi propio idioma, la cual desconocía por completo. (Carolina, cuestionario, 06/08/2013)

\section{Logros}

Las diferentes actividades desarrolladas durante el curso y las reflexiones realizadas tuvieron un efecto en la formación de los futuros docentes; particularmente en el desarrollo de las diferentes actitudes, aptitudes y saberes propuestos por MontagneMacaire (2008) presentados en la Introducción y que retomo para el análisis.

Con respecto a los dos primeros ítems (actitudes de aceptación positiva, de interés por la diversidad lingüística y cultural; y, saberes sobre las lenguas y las culturas), los estudiantes demostraron actitudes de interés por otras lenguas y culturas no solo extranjeras sino especialmente de nuestro propio país. El descubrimiento de la variedad lingüística del país representada en sus lenguas indígenas y criollas amplió la visión de los estudiantes sobre la situación lingüística del país y sobre la necesidad de valorarla, protegerla y difundirla. Así lo dejan entrever los siguientes comentarios:

Creo que [el curso] me dio herramientas, y abrió mis ojos. Uno como docente de lenguas extranjeras tiene la tendencia a cerrarse en precisamente eso, las lenguas extranjeras $y$, afortunadamente, pude conocer la importancia de las lenguas de mi propio país, de las culturas - esas culturas lo hacen a uno! [sic]. (Diana, cuestionario, 08/06/2013)

[El curso] tuvo una fuerte influencia ya que nos quita la idea de la cabeza del uso del inglés y del francés como lenguas únicas. Más que conocer es apreciar y difundir los otros miles de lenguas que habla y comparte la humanidad. (Mauricio, cuestionario, 10/08/2013)

[M]i concepción de las lenguas cambio [sic] totalmente, ya que me he vuelto consciente de la importancia que tienen todas y cada una de lenguas que se hablan alrededor del mundo y de la importancia que tiene generar acciones para conservarlas. Anteriormente, mi objetivo era difundir el aprendizaje del francés y del inglés, ya quiero acercarme a otras lenguas y difundir la importancia de una educación al plurilingüismo. (Iván, cuestionario, 10/08/2013)

El testimonio anterior muestra que el curso no solo ayudó a concientizar sobre la importancia de valorar las lenguas, sino que además impulsó a los estudiantes a pensar en acciones concretas que ellos podrían tomar en sus clases de lengua extranjera para favorecer la reflexión sobre estos temas entre sus estudiantes.

Con respecto al punto tres (aptitudes para: utilizar varias lenguas con fines comunicativos; comunicar parcialmente en la ausencia de un código lingüístico común; y saber identificar lenguas y culturas desconocidas), la reflexión sobre sus propios repertorios lingüísticos es una muestra de que los estudiantes pueden utilizar varias lenguas con fines comunicativos. Asimismo, la participación en una sesión de la plataforma Galanet les permitió acercarse a lenguas desconocidas para algunos como el rumano, el portugués y el italiano. Aunque el objetivo del curso no era aprender ninguno de esos idiomas, el acercamiento a ellos por medio de la plataforma Galanet y de diversas actividades de despertar a las lenguas les ayudó a enriquecer su competencia plurilingüe y pluricultural. Los estudiantes concluyeron que ambos enfoques (intercomprensión y despertar a las lenguas) podrían implementarse en nuestro contexto colombiano. Algunos se aventuran a proponer que dichos enfoques podrían utilizarse para promover el conocimiento de las lenguas propias de Colombia, como lo manifestó Diana:

\footnotetext{
Por otra parte, la experiencia en Galanet —aunque corta y limitada por diferentes circunstanciasme pareció muy enriquecedora. Creo que como docente podría hacerme de diferentes estrategias para abrirle [sic] las puertas a mis estudiantes [...] ante la gran posibilidad de lenguas a explorar, de culturas e [sic] vivir y sentir. (Diana, cuestionario, 08/06/2013)
} 
Miremos ahora algunos logros en el ámbito académico. En la más reciente versión del curso, el trabajo final consistía en preparar una clase de inglés o francés en la cual los estudiantes debían integrar algunos de los elementos aprendidos durante el curso. Los trabajos de los estudiantes reflejaron en su mayoría una amplia apropiación de los conceptos y su integración en la preparación de clases. En el anexo 1, dos futuros docentes proponen una clase de francés en la cual los estudiantes descubren algunas características de los pueblos indígenas del país por medio de actividades inspiradas en los enfoques intercultural y de despertar a las lenguas.

Igualmente, las reflexiones de algunos estudiantes permiten ver la apropiación de conceptos en su rol como maestros de lenguas.

(Les enseignants de langues) doivent avoir une connaissance très vaste sur le contexte national, la culture qui nous entoure et les différentes langues indigènes qu'il y a en Colombie parce que la seule manière d'avoir une population plurilingue c'est en commençant par la reconnaissance de ses propres racines culturelles. (Marta, reflexión final, 20/02/2012)

Et que doit-on faire pour préserver nos langues indigènes ? On peut commencer par lécole, [...] c'est dans ce point-là que les langues parlées tout au long du territoire national peuvent être l'objet d'un éveil aux langues. (Sandra, texto de reflexión sobre las lenguas indígenas de Colombia, 19/09/2011)

Asimismo, los estudiantes evaluaron como muy positivo el aporte de este curso a su formación docente. Así lo explican los siguientes estudiantes:

Este curso tuvo mucho aporte a mi formación como docente ya que pude repensar el papel pedagógico y su contribución en la enseñanza de la cultura indígena como parte de la formación plurilingüe con miras a construir una educación más equitativa e inclusiva en Colombia. (Carolina, cuestionario, 06/08/2013)

Aprendí que no se debe menospreciar ningún idioma ya que son parte del patrimonio de la humanidad. También aprendí a valorar el patrimonio lingüístico local. Aprendí que a través de una actividad d'eveil aux langues se pueden promover valores como el respeto, la tolerancia. (Víctor, cuestionario, 06/08/2013)

Me gustaría mucho introducir a los estudiantes que tenga en un futuro a este tema. Quisiera utilizar la información sobre las comunidades indígenas aquí en Colombia y en otros países como una manera de motivar a los estudiantes a aprender nuevas lenguas y culturas. Para abrirles los ojos al mundo... aunque sea un poco y dejarles ver que afuera hay mucho por conocer. (Estefanía, cuestionario, 07/08/2013)

Estos testimonios muestran una apertura en el pensamiento de los estudiantes sobre su rol de maestros de lenguas y en la construcción de su identidad profesional. Aunque saben que en el futuro enseñarán quizás una sola lengua extranjera, son conscientes de que en sus clases podrán promover la diversidad lingüística.

Con respecto a la identidad, los hallazgos confirman que esta es dinámica y en evolución constante, como se señaló en el marco teórico. De hecho, a través de las discusiones en clase y de los textos escritos por los estudiantes, fue posible observar cómo los maestros en formación reflexionaron sobre sus diferentes facetas identitarias. En el ámbito de la identidad lingüística, por ejemplo, el curso permitió una reflexión sobre lo que implica hablar una lengua en tanto que lengua extranjera. Se desmitificaron asuntos como la importancia de tener a un hablante nativo como modelo ideal, en consonancia con la visión de las lenguas dentro de la didáctica del plurilingüismo, y el posicionamiento identitario que alguien puede mostrar en las huellas del "acento" de la lengua materna al expresarse en una lengua extranjera. Todas estas reflexiones contribuyen a la formación de la identidad del maestro intercultural que buscamos en nuestro programa y en muchos otros programas del país; un maestro reflexivo y consciente de las particularidades de sus estudiantes. Si bien no fue posible observar las prácticas pedagógicas de los maestros en formación para analizar la 
Universidad Pedagógica Nacional

Facultad de Humanidades

integración de los conceptos estudiados, sí es posible afirmar que dichos estudiantes son más conscientes de los temas trabajados. Este es un primer paso para integrar los conceptos a las prácticas pedagógicas, pues si no se es consciente de la diversidad presente en nuestro contexto local y nacional, no es posible implementar acciones pedagógicas que favorezcan su conocimiento y valoración en las aulas.

A la postre, es importante destacar que la visión intercultural del curso enriqueció la formación de los futuros maestros. Particularmente, el encuentro con el Otro, representado, por ejemplo, en los miembros de las comunidades indígenas con quienes los estudiantes tuvieron contacto, permeó la visión que un maestro de lenguas puede tener. En efecto, no se trata solo de un maestro que se dedica a enseñar aspectos lingüísticos sino de un maestro que puede contribuir al conocimiento y la valoración del Otro en su clase. En este sentido, fue significativo descubrir que un maestro de lenguas puede enriquecer su práctica pedagógica a partir del conocimiento y de otras formas de ver el mundo que pueden aportar los miembros de comunidades ancestrales.

\section{Conclusiones}

La experiencia de cuatro años de trabajo dictando este curso me ha permitido reafirmar la necesidad de buscar espacios en los programas de formación de maestros de lenguas para reconocer nuestra diversidad y continuar construyendo nuestra propia identidad. Desafortunadamente, desde la concepción de los programas, buscamos en muchas ocasiones enfocarnos en lo foráneo olvidando que desde una visión intercultural siempre debemos empezar por reconocernos a nosotros mismos, saber quiénes somos, de dónde venimos.

Los 65 estudiantes que han completado el curso de "Introducción al Plurilingüismo" hasta la fecha han reconocido como significativo el aporte del curso a su formación como sujetos plurilingües y como docentes de lenguas extranjeras. Dichos estudiantes pueden considerarse como sujetos plurilingües según las perspectivas presentadas en este texto y otras como la de Kramsch (2009).
Los datos analizados permiten ver cambios en las percepciones de los maestros en formación y sobre todo una mayor apertura a la diversidad cultural y lingüística de nuestro país. Esto se logró gracias a la variedad de actividades y reflexiones propuestas en el curso. No obstante, es preciso señalar que el análisis se hizo desde los discursos de los sujetos, mas no desde una observación de sus prácticas como docentes. En este sentido, el no haber llegado al terreno del aula para ver cómo lo trabajado en el curso podría impactar las acciones pedagógicas de los maestros en formación constituye una limitación de este trabajo.

Sería imposible concluir que fue exclusivamente gracias a este curso que los estudiantes desarrollaron la competencia plurilingüe y pluricultural. Es más, sería incorrecto afirmar que los estudiantes ya han desarrollado dicha competencia plenamente pues, como se explicó en el marco teórico, se trata de un concepto evolutivo y por ende no es una competencia que se pueda desarrollar solamente en un curso sino que se enriquece a lo largo de la vida. El itinerario de formación que los estudiantes han seguido a lo largo del programa al aprender dos idiomas y las trayectorias de vida de cada uno - de contacto con otras lenguas y culturas- han sido fundamentales en el desarrollo de dicha competencia. El curso permitió generar conciencia sobre ella y enriquecerla. También es importante explicar que, aunque se trate de un concepto propuesto en Europa y para Europa, se ha adaptado a las particularidades de nuestro contexto. En otras palabras, la competencia plurilingüe y pluricultural de nuestros estudiantes debe verse permeada por el acercamiento a las lenguas y culturas autóctonas de nuestro país y no solo a las extranjeras.

Una formación en el plurilingüismo es pertinente, incluso necesaria, para los estudiantes que aprenden dos idiomas extranjeros de forma simultánea y que se preparan para enseñarlos. Dicha reflexión les permite no solo comprender y valorar su propia situación lingüística, sino acercarse a nuevas visiones de la didáctica de las lenguas concebida como una didáctica del plurilingüismo. 
Este enfoque es válido en un momento histórico en el cual se sobredimensiona la importancia de una sola lengua extranjera (inglés) en detrimento de las otras, particularmente en las políticas del Gobierno colombiano en lo que a la enseñanza de lenguas extranjeras se refiere. Muchos, en otras latitudes, comienzan a cuestionar que el inglés sea la única lengua favorecida en la comunicación internacional y ofrecen otras alternativas desde el plurilingüismo, por ejemplo, con los enfoques plurales. Igualmente, los actuales movimientos de revitalización de lenguas minoritarias y minorizadas cuestionan la importancia de una sola lengua. Estas razones deberían seguir impulsando acciones a favor de la promoción de la diversidad lingüística y cultural en contextos monolingües y en la formación de maestros de lenguas.

Los resultados favorables que esta propuesta ha tenido dentro del plan de formación de maestros de lenguas de la Universidad de Antioquia han llevado a tomar la decisión de incluir este curso, con algunas adaptaciones, como obligatorio en la nueva versión del programa, en la cual se trabaja desde hace algunos años. Por ser un tema de reciente discusión en nuestro contexto, es necesario continuar su promoción y discusión, y analizar los efectos de este tipo de reflexiones en las prácticas de los maestros de lenguas extranjeras.

\section{Referencias bibliográficas}

Agudelo, J. (2007). An intercultural approach for language teaching: Developing critical cultural awareness. Ikala, Revista de Lenguaje y Cultura, 12 (18), 185-217.

Albarello, L. (2003). Apprendre à chercher, l'acteur social et la recherche scientifique. Bruxelles, Belgique: De Boeck.

Arismendi, F. (2011). Interactions en ligne et interculturalité: le cas de Galanet, plate-forme consacrée à l'interaction en langues romanes. Lenguaje, 39 (1), 165-196.

Bernaus, M.; Andrade, A.; Kervran, M.; Murkoswa, A. \& Trujillo, F. (2007). La dimension plurilingue et pluriculturelle dans la formation des enseignants de langues. Kit de formation. Strasbourg: Éditions du Conseil de l'Europe.
Bloomfield, L. (1935). Language. Nueva York: Henry Holt.

Candelier, M. (2006). Léveil aux langues - une proposition originale pour la gestion du plurilinguisme en milieu scolaire - Contribution au Rapport mondial de l'Unesco Construire des Sociétés du Savoir. En D. Cunningham, R. Freudenstein \& C. Odé, (comp.), Language teaching: A worldwide perspective - celebrating 75 years of FIPLV (pp. 145-180). Belgrave: FIPLV.

Candelier, M. (2008). Approches plurielles, didactique du plurilinguisme: le même et l'autre. Les Cahiers de l'Acedle, 5 (1), 65-90.

Cárdenas, M. (2006). Bilingual Colombia: Are we ready for it? What is needed? Ponencia presentada durante el 19th Annual English Australia Education Conference, realizado en Perth, Australia.

Castellotti, V. (2006). Une conception plurielle et intégrée de l'enseignement des langues - principes, modalités, perspectives. Les Cahiers de l'Acedle, 2, 319-331.

Castellotti, V. \& Moore, D. (2011). La compétence plurilingue et pluriculturelle: genèse et évolutions d'une notion-concept. En P. Blanchet \& P. Chardenet (comp.). Guide pour la recherche en didactique des langues et des cultures (pp. 241-252). Paris: éditions des archives contemporaines.

Colombia (1991). Constitución Política, Bogotá: Legis.

Concejo de Medellín (2013). Acuerdo 89 de 2013 “Por medio del cual se adoptan las Bases de la Política Pública de Procesos de Enseñanza de Lenguas Extranjeras en la Ciudad de Medellín”. Disponible en http://www.concejodemedellin.gov.co/concejo/ m21_gallery/23709.pdf

Congreso de Colombia (2013). Ley 1651 del 12 de julio de 2013. Disponible en http://goo.gl/irhA2x.

Coste, D. (2010). Diversité de plurilinguismes et formes de l'éducation plurilingue et interculturelle. Les Cahiers de l'Acedle, 7 (1), 141-165.

Degache, C. (2006). Didactique du plurilinguisme. Travaux sur l'intercompréhension et l'utilisation des technologies pour l'apprentissage des langues. Recuperado de www.galanet.eu/publication/fichiers/ HDR2006_DegacheC.pdf

Dendinger, M. (2000). How to organize a focus group. Meetings and conventions. Disponible en http://goo. gl/EQjf3w.

Escudé, P. \& Janin, P. (2010). Le point sur l'intercompréhension clé du plurilinguisme. Paris: CLE International. 
Universidad Pedagógica Nacional

Facultad de Humanidades

Galindo, A. \& Moreno, L. (2008). Producción argumentativa escrita - lengua materna - en contextos educativos bilingües y monolingües: grados $6^{\circ}, 7^{\circ}$ y $8^{\circ}$. Ikala, Revista de Lenguaje y Cultura, 13 (19), 145-169.

García, J. (2014). La gente te define por la lengua que hablas, si hablas mucho criollo van a decir que no eres culto. Reflexiones en torno a la identidad lingüística en hablantes de inglés criollo, inglés estándar y español. Lenguaje, 42 (1), 145-170.

González, A. (2009). Reflexiones sobre el bilingüismo en Colombia. El Educador, 7 (2), 24-39.

González de Pérez, M. (2012). Los gitanos y su lengua. En C. Patiño Rosselli, \& J. Bernal, (comp.). El lenguaje en Colombia. Tomo I: Realidad lingüística de Colombia (pp. 738-757). Bogotá: Instituto Caro y Cuervo.

González de Zárate, M. (2007). Por un plurilingüismo desde la escuela primaria. Magisterio, 25, 58-60.

Grosjean, F. (1982). Life with two languages: An introduction to bilingualism. Cambridge, Mass: Harvard University Press.

Grosjean, F. (2010). Bilingual, life and reality. Cambridge: Harvard University Press.

Guerrero, C. (2008). Bilingual Colombia: What does it mean to be bilingual within the framework of the National Plan of Bilingualism? Profile, 10, 27-45.

Guerrero, C. (2009). Language policies in Colombia: The inherited disdain for our native languages. How, A Colombian Journal for Teachers of English, 16, 11-24.

Guerrero, C. (2012). La enseñanza del inglés como L2: limitaciones y alcances para la formación ciudadana. Magisterio, 58, 44-50.

Herazo, J.; Jerez, S. \& Lorduy, D. (2012). Opportunity and incentive for becoming bilingual in Colombia: Implications for Programa Nacional de Bilingüismo. Íkala, Revista de Lenguaje y Cultura, 17 (2), 199-213.

Instituto Cervantes (2002). Marco Común Europeo de Referencia para las Lenguas: Aprendizaje, enseñanza, evaluación. Recuperado de https://www.forem.es/ assets/files/mcerl.pdf.

Kastersztein, J. (1990). Les stratégies identitaires des acteurs sociaux: approche dynamique des finalités. En C. Camilleri et al. (comp.) Les stratégies identitaires (pp. 27-41). Paris: PUF.

Klett, E. (2014). Formations des enseignants et plurilinguisme: quels moyens peut-on se donner? Revue de la
Sapfesu, Sociedad Argentina de Profesores de Francés de la Enseñanza Superior y Universitaria, 37, 80-90.

Klett, E. (2011). Aprendizaje de lenguas y plurilingüismo. Trabajo presentado en el II Congreso de enseñanza de español como lengua segunda y extranjera. Universidad del Salvador, Buenos Aires, 13-15 de abril de 2011. Recuperado de http://www.salvador. edu.ar/vrid/ead/Klett.pdf

Kramsch, C. (2009). The multilingual subject. New York, NY: Oxford University Press.

Lagarde, C. (2008). Identité, langue et nation. Qu'est-ce qui se joue avec les langues? Canet: Trabucaire.

Landaburu, J. (2012). Colombia: Tesoro indígena colombiano en riesgo. Recuperado de http://www.eltiempo. com/archivo/documento/CMS-11983681

Macnamara, J. (1967). The bilingual's linguistic performance-a psychological overview. Journal of Social Issues, 23 (2), 58-77.

Mejía, A. M. de (2002). Power, prestige and bilingualism: International perspectives on elite bilingual education. Clevedon, UK: Multilingual Matters Ltd.

Mejía, A. M. de (2004). Bilingual Education in Colombia: Towards an Integrated Perspective. Bilingual Education and Bilingualism, 7 (4), 381-397.

Mejía, A. M. de (2006). Bilingual education in Colombia: Towards a recognition of languages, cultures and identities. Colombian Applied Linguistics Journal, $8,152-168$

Mejía, A. M. de (2007). Visiones del bilingüismo y de la educación bilingüe en Colombia. Magisterio, 25, 36-38.

Ministerio de Educación Nacional Nacional (MEN). (2014). Lineamientos de calidad para las licenciaturas en educación (programas de formación inicial de maestros). Recuperado de http://goo.gl/Fzt1Yh.

Ministerio de Educación Nacional (MEN). (1994). Ley General de Educación (Ley 115 del 8 de febrero de 1994). Recuperado de http://goo.gl/t02Y8p

Miranda, I. (2012). Insights on bilingualism and bilingual education: A sociolinguistic perspective. Ikala, Revista de Lenguaje y Cultura, 17 (3), 263-272.

Montagne-Macaire, D. (2008). D'une didactique de langues à une didactique des plurilinguismes? Réflexions pour la recherche. Les Cahiers de l'Acedle, 5 (1), 3-40.

Moore, D. (2006). Plurilinguismes et école. Paris: Editions Didier. 
Moore, D. \& Castellotti, V. (2001). Comment le plurilinguisme vient aux enfants. En V. Castellotti (Comp.). D’une langue à d'autres (pp. 151-189). Rouen: Presses Universitaires de Rouen.

Ordóñez, C. (2012). Educación para el bilingüismo y aprendizaje de maestros: comprensión del desempeño auténtico en la acción de cambiar prácticas pedagógicas. Folios, 36, 3-22.

Rengifo, D. (2011). Éveil aux langues et au langage en contexte monolingue. Trabajo presentado en el xxième Congrès National de l'Acolprof "La réintroduction du français en Colombie: du repli au renouveau”». Pontificia Universidad Javeriana, Bogotá, 12, 13 y 14 de diciembre del 2011.

Riley, P. (2003). Le "linguisme"- multi- poli- pluri ? Points de repère terminologiques et sociolinguistique. Le Français dans le monde, recherche et applications numéro spécial, juillet 2003, 8.-17.

Sabatier, C. (2010). Plurilinguismes, représentations et identités: des pratiques des locuteurs aux définitions des linguistes. NPSS, 6 (1), 125-157.

Sánchez, A. C. \& Obando, G. V. (2008). Is Colombia ready for "Bilingualism"? Profile, 9, 181-196.

Simon, D.-L. (2005). Faire chanter les voix/voies de la pluralité : un nouveau défi pour l'école. En M.-A. Mochet, M.-J. Barbot, V. Castellotti et al. Plurilinguisme et apprentissages (pp. 133-142). Paris: L'Harmattan.

Tapiero, O. (2014). Promoción de la diversidad de la lengua en contextos de homogeneidad. Lenguaje, 42 (2), 231-256.

Tassara, G. \& Villalón, C. (2014). La intercomprensión de lenguas latinas: una herramienta para el desarrollo del plurilingüismo en Chile. Colombian Applied Linguistics Journal, 16 (2), 277-290.

Usma, J. (2009). Education and language policy in Colombia: Exploring processes of inclusion, exclusion, and stratification in times of global reform. Profile: Issues in Teachers' Professional Development, 11, 123-141.

Valencia, S. (2007). El bilingüismo y los cambios en políticas y prácticas en la educación pública en Colombia: un estudio de caso. Ponencia presentada en el II Simposio Internacional de Bilingüismo y Educación Bilingüe en América Latina, Bogotá. Recuperado de https://goo.gl/2E45Iw
Van der Maren, J.-M. (2003). La recherche appliquée en pédagogie. Des modèles pour l’enseignement. Bruxelles, Belgique: De Boeck Université.

\section{Cibergrafía}

Galanet: www.galanet.eu

\section{Anexo 1}

Ejemplo de plan de clase realizado por dos estudiantes en el semestre 2014- ${ }^{15}$

Durée: 1 h30 Niveau: Adolescents ou adultes ayant un niveau $\mathrm{A} 2+$ ou $\mathrm{B} 1$

\section{Objectif général:}

- Sensibiliser les apprenants à l'existence des langues et communautés indigènes en Colombie.

\section{Objectifs Spécifiques:}

- Réfléchir sur les stéréotypes associés aux indigènes en Colombie.

Relier une des langues connues, soit le français soit l'espagnol, avec une langue indigène.

Objectifs Opérationnels:

- Létudiant est capable de répondre aux questions à partir de la lecture du document déclencheur.

- Létudiant est capable d'associer les mots d'une langue indigène à la langue Française.

- Létudiant est capable d'assembler un puzzle afin de repérer de l'information pour une petite présentation.

- Létudiant est capable de présenter l'information essentielle d'une communauté indigène colombienne.

Avant la lecture (Mise en Commun à la fin)

Tâche 1 (Individuelle - 10 minutes)

1. Si vous regardez le mot "Indigène" quels sont les mots qui viennent à votre esprit? Vous allez remplir un cercle de concepts (6 concepts minimum).

15 Este plan de clase fue elaborado por los estudiantes Yesica Alejandra Moreno Moreno y Mario Pinto Nocua en el semestre 2014-I y se reproduce aquí con su previa autorización. 


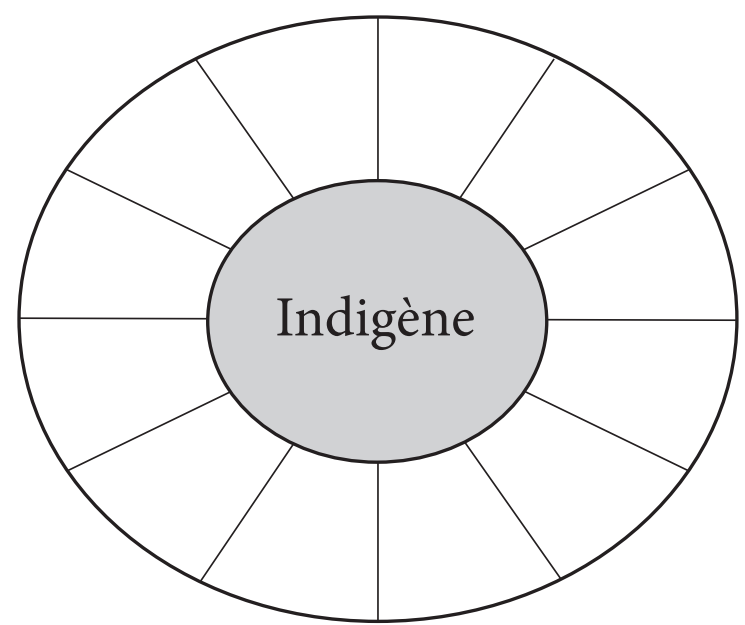

2. D’après ce que vous connaissez, combien de langues on parle en Colombie?

3. Combien de langues indigènes il y a en Colombie?

a. $5-10$

b. $10-20$

c. $30-50$

d. Plus de 60

Pendant la lecture (Mise en Commun à la fin de l'activité)

Tâche 2 (Petits Groupes - 20 minutes)

Consigne à l'oral

1. Organisez le texte dans l'ordre que vous croyez cohérent. (5 minutes)

2. Lisez le texte original (dans l'ordre correct), après la première lecture, faites une liste de langues indigènes que vous rappelez de la lecture. (10 minutes)

3. Lisez le texte pour la deuxième fois et corrigez vos réponses si nécessaire. (5 minutes)
La Colombie possède l'un des héritages indigènes les plus variés au monde. Il est constitué d'un éventail de cultures, de langues, de structures sociales et de modes de vie. Selon le recensement de 2005, environ 1,4 millions de personnes indigènes vivent en Colombie, soit approximativement $3,4 \%$ de la population totale du pays.

En Colombie, au moins 85 tribus indigènes, utilisent plus de 60 langues natives qui appartiennent à 22 familles linguistiques ; 65 langues indigènes, deux langues d'ascendance africaine (le créole et le palenquera) et le Romani des Gitans.

Parmi les langues indigènes parlées aujourd'hui en Colombie, seulement trois comptent plus de 50000 locuteurs: le wayú, le paez et l'embera. Huit comptent entre 10000 et 50000 locuteurs: le guahibo (ou sikuani), le guambiano, l'arhuaco (ou ika), l'inga, le ticuna, le tucano, le berce, le piaroa.

Moins d'une dizaine d'autres langues dénombrent entre 5000 et 10000 locuteurs: le cuaiquer (ou awá), le kogui, le waunana, le puinave, le wuitoto, le curripaco, le piapoco, le yaruro et le yuco. Une douzaine de langues comptent entre 1000 et 5000 locuteurs: le tunebo (ou úwa), le cubeo, le camsá, le wiwa, le bari, le cofán, le cuiba, le coreguaje, le sáliba, le guayabero et le yagua.

Toutes les autres langues comptent moins de 1000 locuteurs: totoró, barasano, desano, wanano, piratapuyo, achagua, andoke, bará, bora, cabiyarí, carapana, carijona, chimila, colit, hitnu, macuna, cacua, nukak, hupda, yuhup, miraña, muinane, nonuya, ocaina, pisamira, siona, siriano, tanimuka, tariano, tatuyo, tinigua, tuyuca, yucuna, yurutí, etc. La plupart de ces langues sont donc en voie d'extinction. Les linguistes colombiens ont établi une liste de 20 langues « en danger potentiel ", 11 langues "en danger ", quatre langues « en sérieux danger »et plusieurs langues «moribondes», toutes parlées par moins de 100 locuteurs, parfois deux ou trois.

Adapté de: http://www.axl.cefan.ulaval.ca/ amsudant/colombie.htm 


\section{Après la lecture (Mise en Commun)}

Tâche 3 (Toute la classe - 10 minutes)

Questions de base à partir de la lecture du document (à loral)

Comment avez-vous trouvé le document?

Vos réponses dans la tâche 1(2-3) sont-elles correctes?

Connaissez-vous ces langues indigènes?

Que veut dire l'auteur par la phrase langues en danger potentiel et langues en sérieux danger?

Les langues indigènes, sont-elles importantes pour la Colombie?

Qu'implique pour la Colombie le fait de perdre une langue indigène?

\section{Tâche 4 (Petits Groupes - 10 minutes)}

Essayez d'associer les mots suivants. Il s'agit du nom des animaux dans la langue Kogui, qui appartient à l'ethnie Kogui de la Sierra Nevada de Santa Marta.

\begin{tabular}{|c|c|}
\hline Kogui & Français \\
\hline Miku __ & a) chauve-souris \\
\hline Sugi ___ & b) Hibou \\
\hline Pio ___ & c) Ara \\
\hline Kaklabe __ & d) Colibri \\
\hline Kuakuldu __ & e) Chien \\
\hline Singsi ___ & f) Caïman \\
\hline Melkua __ & g) Souris \\
\hline Sinduldi ___ & h) Araignée \\
\hline Wauhu __ & i) Poisson \\
\hline Takbi __ & j) Cerf \\
\hline Tultuga ___ & k) Oiseau \\
\hline Kaimanu ___ & I) Ouistiti \\
\hline Waxa ___ & m) Jaguar \\
\hline Nuba __ & n) Tortue \\
\hline Niuwishy ___ & o) Serpent \\
\hline
\end{tabular}

Questions de base à partir de l'exercice d'association (à l'oral)

- Quel mot vous avez trouvé le plus facile? Lequel le plus difficile? Pourquoi?

- Trouvez-vous quelque relation entre cette langue et l'espagnol?

\section{Tâche 5 (Petits Groupes - 30 minutes)}

Vous recevez un puzzle à résoudre. Il contient une image avec une expression artistique (artisanat) propre d'une communauté indigène de notre pays. De la même manière, le puzzle contient un texte avec de l'information sur quelques aspects généraux de l'ethnie. Après organiser les pièces, lisez le texte et préparez une petite présentation pour partager de l'information avec vos camarades.

N.b.: Le puzzle est fait à partir d'une photo représentant un icône indigène comme une mola ou un vêtement traditionnel. 